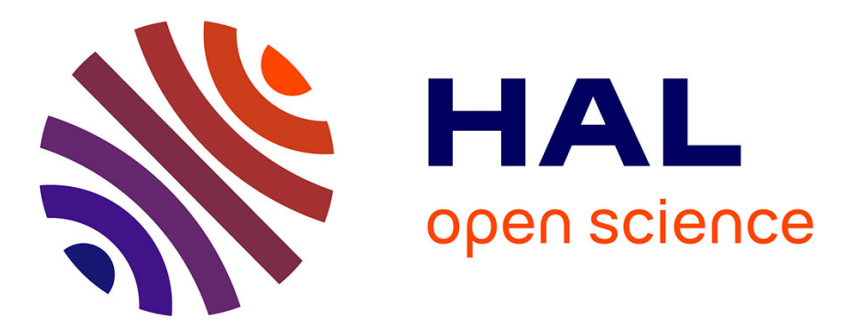

\title{
Méthode de mesure de l'énergie d'ancrage d'un nématique. Application a l'ancrage sur une paroi non traitée
}

\author{
J. Sicart
}

\section{- To cite this version:}

J. Sicart. Méthode de mesure de l'énergie d'ancrage d'un nématique. Application a l'ancrage sur une paroi non traitée. Journal de Physique Lettres, 1976, 37 (1), pp.25-27. 10.1051/jphyslet:0197600370102500 . jpa-00231223

HAL Id: jpa-00231223

https://hal.science/jpa-00231223

Submitted on 1 Jan 1976

HAL is a multi-disciplinary open access archive for the deposit and dissemination of scientific research documents, whether they are published or not. The documents may come from teaching and research institutions in France or abroad, or from public or private research centers.
L'archive ouverte pluridisciplinaire HAL, est destinée au dépôt et à la diffusion de documents scientifiques de niveau recherche, publiés ou non, émanant des établissements d'enseignement et de recherche français ou étrangers, des laboratoires publics ou privés. 


\title{
Classification \\ Physics Abstracts 7.130 \\ MÉTHODE DE MESURE DE L'ÉNERGIE D'ANCRAGE D'UN NÉMATIQUE. APPLICATION A L'ANCRAGE SUR UNE PAROI NON TRAITÉE
}

\author{
J. SICART \\ Groupe de Dynamique des Phases Condensées (*) \\ Laboratoire de Minéralogie et Cristallographie, Université des Sciences \\ et Techniques du Languedoc, Place Eugène Bataillon, 34060 Montpellier, France
}

(Reçu le 16 juillet 1975, révisé le 15 octobre 1975, accepté le 10 novembre 1975)

\begin{abstract}
Résumé. - Nous proposons une méthode simple de détermination de l'énergie d'ancrage de torsion d'une lamelle cristal-liquide nématique. Nous avons appliqué cette méthode au cas d'ancrages très faibles (lames de verre non traitées). L'échantillon nématique est placé entre deux lames de verre dont une seulement est traitée par frottement. En tournant de $\pi / 2$ la lame non traitée, on impose une torsion au nématique. Si on applique maintenant un champ magnétique parallèle à la direction de frottement, on observe une rotation du directeur sur la lame non traitée.

Les conditions d'équilibre permettent alors de déterminer l'énergie d'ancrage de torsion sur la surface.
\end{abstract}

\begin{abstract}
An easy method to determine the anchoring energy of twist for a nematic liquidcrystal layer is presented. We have applied this method to the case of very weak anchoring (untreated sheets of glass). The nematic is introduced between two glass plates; only one is treated by rubbing. We twist the nematic by turning the non-rubbed plate by $\pi / 2$. If one now applies a magnetic field along the rubbing direction, the director direction on the untreated plate changes. The equilibrium conditions then allow determination of the anchoring energy of twist on the surface.
\end{abstract}

1. Introduction. - L'étude de l'ancrage sur les parois d'un nématique a fait l'objet de plusieurs travaux en particulier relatifs aux effets électrooptiques de la distorsion d'une lamelle nématique sous champ magnétique $[1,2]$. Des mesures d'énergie libre d'adhésion par ancrage physico-chimique ont été faites à partir de calculs de densités et moments dipolaires [3]. Des mesures à partir d'étude de lignes de désinclinaison peuvent donner également des estimations d'énergie d'ancrage superficielle sur lames frottées $[4,5,6]$.

Il faut distinguer selon les cas l'ancrage de type planaire ou héméotrope. Nous proposons ici d'effectuer des mesures d'énergie d'ancrage de torsion de préparations nématiques planaires.

La méthode que nous proposons exige des champs magnétiques élevés pour des énergies d'ancrage fortes. $\mathrm{Ne}$ disposant pas de champs magnétiques élevés, nous avons cherché à réaliser des ancrages faibles. C'est le cas de l'ancrage naturel des tolanes sur une lame de verre n'ayant subi aucun traitement mécanique.

(*) Laboratoire associé au C.N.R.S. $n^{\circ} 233$.
Nous avons en effet observé que si l'on place un mélange équimoléculaire de tolanes entre une lame frottée et une lame non traitée, et que l'on fasse ensuite tourner la lame non traitée de $\pi / 2$, le plan de polarisation de la lumière transmise subit également une rotation de $\pi / 2$.

Cet effet peut probablement être attribué à une adsorption des molécules de tolanes à la surface de la lame non traitée.

Une expérience similaire tentée sur du M.B.B.A. n'a pas donné ce résultat de manière reproductible, ce qui tendrait à prouver que l'ancrage naturel du M.B.B.A., s'il existe, est beaucoup plus faible.

Ces mesures d'énergie présentent un intérêt tout particulier vu l'utilisation intensive de nématiques sous torsion dans les dispositifs électro-optiques.

2. Méthode expérimentale. - Les lames non frottées sont des lames floating glass n'ayant subi aucun traitement mécanique. Le seul traitement est un nettoyage par mélange sulfo-chromique suivi d'un rinçage à l'eau distillée.

L'autre face de la préparation est constituée par une lame ayant subi un frottement mécanique suivant le procédé dû à $\mathbf{P}$. Chatelain [7]. 
Le cristal-liquide, nématique à température ambiante, est un mélange équimoléculaire 4-méthoxy 4'-pentyl-tolane et 4-propoxy-4'-heptyl-tolane. Il est placé entre deux cales calibrées en mylar.

Pour mesurer l'ancrage naturel, on effectue une rotation de $\pi / 2$ de la lame supérieure non frottée puis on applique un champ magnétique parallèle à la direction de frottement de la lame inférieure (Fig. 1).

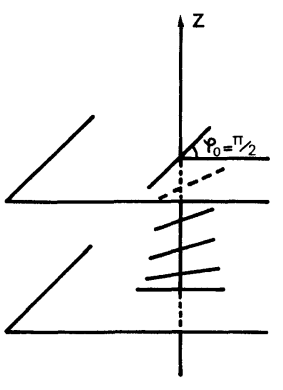

a

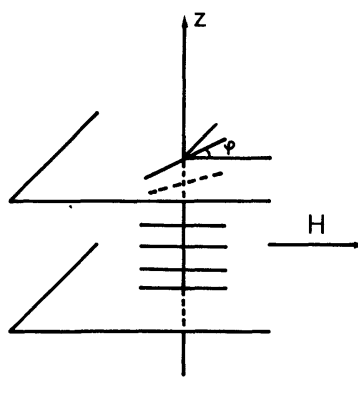

b
FIG. 1. - Lamelle planaire tordue : a) $H=0: \varphi=\varphi_{0}=\pi / 2$; b) $H \neq 0: \varphi<\varphi_{0}$.

Le champ magnétique augmente le couple en surface sur la lame supérieure et a donc tendance à induire une rotation du directeur en surface, donc du plan de polarisation de la lumière émergente. La détection de la vibration émergente se fait à l'aide d'un analyseur circulaire à pénombre de type classique (Fig. 2).

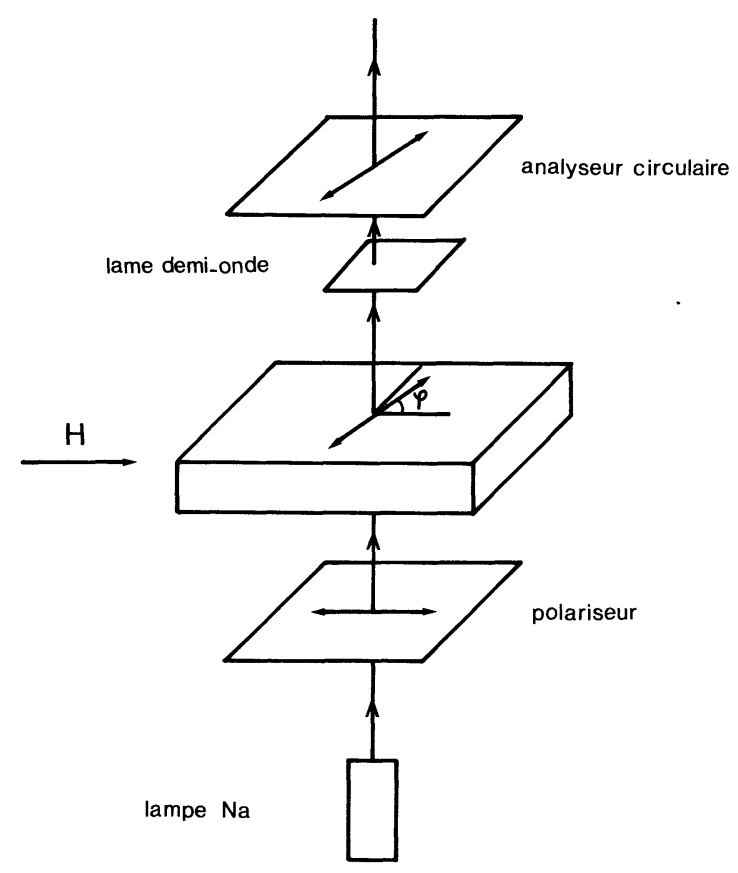

FIG. 2. - Principe du montage expérimental.

3. Conditions d'équilibre. - A l'équilibre, le couple magnétique est contrebalancé par un couple dû à la partie anisotrope de l'énergie d'ancrage en surface. Le couple magnétique et le couple de torsion en volume se déduisent aisément de l'énergie élastique de Frank :

$$
\mathscr{F}=\frac{1}{2} \int_{v}\left\{K_{2}(\mathbf{n} \cdot \operatorname{rot} n)^{2}-\chi_{\mathrm{a}}(\mathbf{n} \cdot \mathbf{H})^{2}\right\} \mathrm{d} V .
$$

La partie anisotrope de l'énergie superficielle a une période de $\pi$.

Nous prendrons donc une énergie de surface de la forme :

$$
\mathscr{F}_{\mathrm{S}}=A-B \cos ^{2}\left(\varphi-\varphi_{0}\right) .
$$

Un calcul variationnel classique conduit à la condition d'équilibre en volume

$$
\frac{\partial \varphi}{\partial Z}=\frac{\sin \varphi}{\xi}
$$

où $\xi$ est la longueur de cohérence magnétique :

$$
\xi=\frac{1}{H}\left(\frac{K_{2}}{\chi_{\mathrm{a}}}\right)^{1 / 2}
$$

et à un couple en surface

$$
\gamma_{\mathrm{s}}=-\frac{K_{2} \sin \varphi}{\xi}
$$

De l'éq. (2) on déduit, pour l'énergie d'ancrage, un couple

$$
\gamma_{\mathrm{a}}=-2 B \cos \left(\varphi-\varphi_{0}\right) \sin \left(\varphi-\varphi_{0}\right) .
$$

Pour $\varphi_{0}=\pi / 2$, l'équilibre des couples conduit à la relation

$$
\sin \left(\varphi_{0}-\varphi\right)=\frac{\left(K_{2} \chi_{\mathrm{a}}\right)^{1 / 2}}{2 B} H
$$

A partir de l'expression (3) on calcule aisément, connaissant $K_{2}$ et $\chi_{\mathrm{a}}$ la valeur de l'énergie d'ancrage $B$. $\varphi_{0}$ et $\varphi$ sont donnés par deux pointés respectivement avant et après l'application du champ magnétique. La mesure de l'anisotropie magnétique $\chi_{\mathrm{a}}=\chi_{\|}-\chi_{\perp}$ a été effectuée par Achard et Sigaud par la méthode du champ magnétique tournant et donne pour notre mélange $\chi_{\mathrm{a}}=\left(1 \times 10^{-7}\right)$ uem-cgs.

On obtient la constante élastique $K_{2}$ en mesurant le champ critique pour la transition de Freedericksz en torsion :

$$
H_{\mathrm{c}}=\frac{\pi}{l}\left(\frac{K_{2}}{\chi_{\mathrm{a}}}\right)^{1 / 2} .
$$

La méthode classique [8] consiste à observer les hyperboles obtenues en lumière convergente d'une préparation planaire entre polariseur et analyseur croisés. Lorsqu'on atteint le champ critique, il se produit une distorsion et une rotation des hyperboles. La mesure conoscopique de $H_{\mathrm{c}}$ pour différentes 
épaisseurs de préparations donne pour notre mélange une valeur de

$$
\frac{K_{2}}{\chi_{\mathrm{a}}}=(6,7 \pm 1) \mathrm{cgs}
$$

c'est-à-dire $K_{2}=\left(6,7 \times 10^{-7}\right)$ cgs.

4. Discussion des résultats. - La figure 3 donne la variation de $\sin \left(\varphi_{0}-\varphi\right)$ en fonction de $H$. De la pente des droites obtenues, on déduit, pour l'énergie d'ancrage en surface les valeurs :

$$
\begin{aligned}
& B_{1}=(5,4 \pm 0,6) 10^{-3} \mathrm{erg} \cdot \mathrm{cm}^{-2} \\
& B_{2}=(4,4 \pm 0,5) 10^{-3} \mathrm{erg} \cdot \mathrm{cm}^{-2}
\end{aligned}
$$

correspondant aux courbes 1 et 2 .

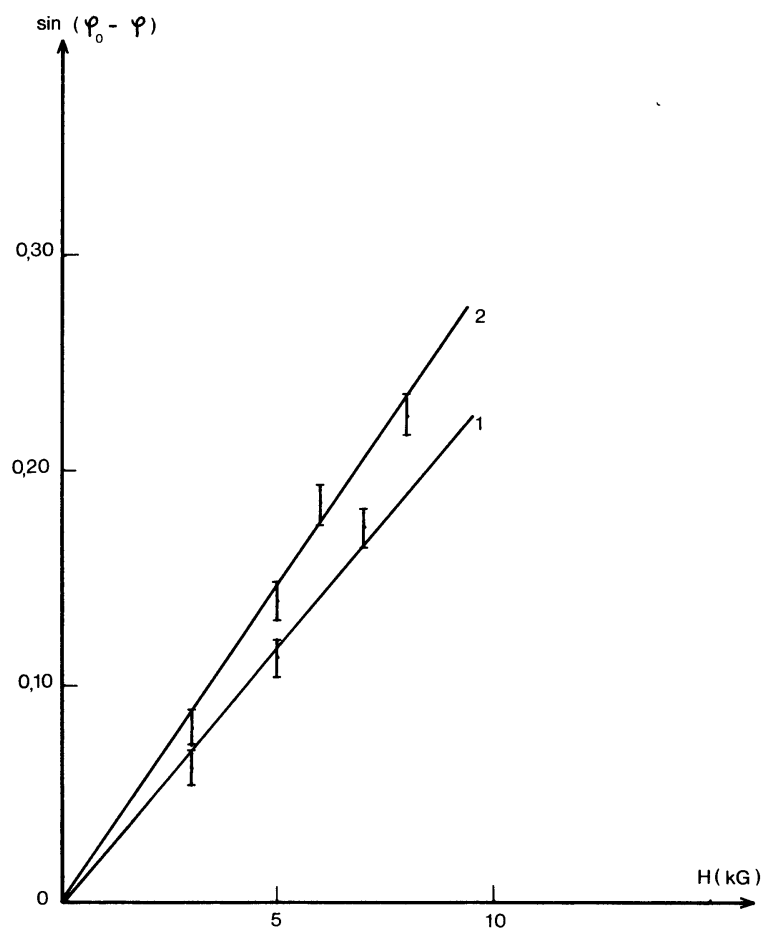

Fig. 3. - Courbes (éq. (3)) donnant la direction du directeur sur la lame non frottée en fonction du champ appliqué. Courbe 1 : épaisseur $50 \mu \mathrm{m}$. Courbe 2 : épaisseur $25 \mu \mathrm{m}$.
Ces deux valeurs sont obtenues sur des préparations différentes. Il faut noter que la torsion magnétique $\left(\varphi_{0}-\varphi\right)$ est reproductible et réversible (si on coupe le champ magnétique, on retrouve la valeur $\varphi_{0}$ ) pour un échantillon donné.

Par contre, deux préparations différentes donnent des résultats légèrement différents, ce qui n'est d'ailleurs pas très étonnant. En effet, l'ancrage naturel doit dépendre de l'histoire de la lame de verre, et en particulier il n'est pas toujours possible d'obtenir un ancrage uniforme sur la totalité de l'échantillon. Il n'en reste pas moins que l'on obtient dans tous les cas un même ordre de grandeur pour l'énergie d'ancrage.

L'épaisseur de l'échantillon ne semble pas influer ' sur les résultats; ceci signifie que le caractère fini de l'ancrage sur la paroi frottée ne joue aucun rôle. Ceci reste vrai tant que la longueur de cohérence magnétique reste petite devant l'épaisseur de l'échantillon.

Enfin il peut être intéressant de comparer les résultats avec ceux obtenus dans le cas de lames frottées, par observations de défauts $[4,5,6]$. Le groupe d'Orsay obtenait des valeurs de l'ordre de

$$
7 \times 10^{-2} \text { erg. } \mathrm{cm}^{-2}
$$

c'est-à-dire environ dix fois plus élevées que les nôtres. Ceci montre l'importance du frottement, et montre aussi que nos résultats sont parfaitement compatibles avec les leurs.

Il faut signaler ici les limitations de la méthode : celles-ci proviennent de la longueur de cohérence magnétique $\xi$. Si $\xi$ est supérieur à l'épaisseur de l'échantillon, le couple magnétique devient négligeable, et la méthode peu sensible. Si au contraire $\xi$ devient très petit devant la longueur d'onde de la lumière, l'approximation adiabatique (lame de Mauguin) utilisée pour la propagation de la lumière n'est plus valable, ce qui limite l'utilisation de la méthode à la mesure d'énergies d'ancrage faibles.

Remerciements. - L'auteur tient à remercier tout particulièrement M. O. Parodi qui est à l'origine de ce travail ainsi que M. F. Achard et J. Sigaud du Centre de Recherche Paul Pascal de Bordeaux-Talence qui ont aimablement effectué les mesures de $\chi_{\mathrm{a}}$.

\section{Bibliographie}

[1] Rapini, A. et Papoular, M., J. Physique Colloq. 30 (1969) C4-54.

[2] Pieranski, P., Thèse 3e cycle, Orsay (1972).

[3] Proust, R. et Ter-Minassian-Saragal, C. R. Hebd. Séan. fcud. Sci. 279 (1974) 615.

[4] Williams, C., Thèse $3^{\mathrm{e}}$ cycle, Orsay (1973).
[5] Williams, C., Vitek, V. et Kleman, M., Solid State Commun. 12 (1973) 581.

[6] Kleman, M. et Williams, C., Phil. Mag. 23 (1973) 721.

[7] Chatelain, P., Bull. Soc. Fr. Mineral. 66 (1943) 105.

[8] Williams, C. et Cladis, P. E., Solid State Commun. 10 (1972) 357. 